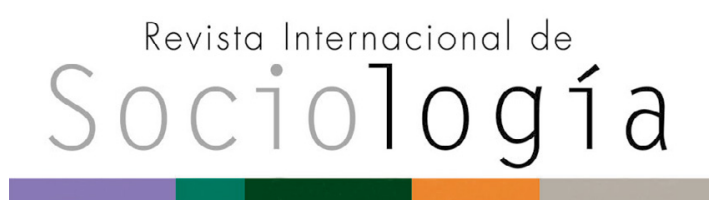

Revista Internacional de Sociología RIS

vol. 79 (2), e186, abril-junio, 2021, ISSN-L:0034-9712 https://doi.org/10.3989/ris.2021.79.2.19.131

\section{ESTRUCTURA FAMILIAR, DETERIORO DEL MERCADO LABORAL Y DESIGUALDAD EN ESPAÑA PARA EL PERÍODO 2008-2017}

\author{
Antonio luis Pérez Corral \\ Universidad de Valladolid \\ antonioluis.perez@uva.es \\ ORCID iD: https://orcid.org/0000-0002-2085-9555 \\ Almudena Moreno Mínguez \\ Universidad de Valladolid \\ almudena@soc.uva.es \\ ORCID iD: https://orcid.org/0000-0002-1839-0508
}

Cómo citar este artículo / Citation: Pérez Corral, A.L. y A. Moreno Mínguez. 2021. "Estructura familiar, deterioro del mercado laboral y desigualdad en España para el período 2008-2017". Revista Internacional de Sociología 79(2):e186. https://doi. org/10.3989/ris.2021.79.2.19.131

\begin{abstract}
Resumen
En este artículo se estudia cómo el deterioro del mercado de trabajo español, a raíz de la crisis económica del año 2008, ha repercutido en la desigualdad laboral y económica de los hogares según la estructura familiar. Para ello, a partir de los datos de la Encuesta de Condiciones de Vida, comparamos la situación de los hogares monoparentales y biparentales en términos de intensidad laboral y bajos ingresos por trabajo. Los principales resultados muestran que, en relación con la intensidad laboral, tanto en el periodo de crisis económica como en el posterior de recuperación ha disminuido la ventaja que las familias monoparentales presentaban con respecto a la biparentales. Por otro lado, aunque los hogares monoparentales registran una mayor probabilidad de experimentar pobreza de ingresos laborales, la diferencia con los hogares biparentales no ha variado a lo largo del periodo de estudio.
\end{abstract}

\section{Palabras claves}

Crisis económica; Desempleo; Precariedad laboral; Hogar

\section{FAMILY STRUCTURE, DETERIORATION OF THE LABOR MARKET AND INEQUALITY IN SPAIN DURING 2008-2017}

Copyright: (c) 2021 CSIC. Este es un artículo de acceso abierto distribuido bajo los términos de la licencia de uso y distribución Creative Commons Reconocimiento 4.0 Internacional (CC BY 4.0).

Recibido: 03/10/2019. Aceptado: 25/09/2020

Publicado: 08/06/2021

\section{Abstract}

This paper examines how the deterioration of the Spanish labor market, following the economic crisis of 2008, has affected household labor and economic inequality according to family structure. To do this, using data from the Living Conditions Survey, we compared the situation of single-parent and two-parent households in terms of labor intensity and low income from work. The main results show that, in relation to labor intensity, both in the period of economic crisis and in the subsequent recovery period, the advantage that single-parent families had with respect to two-parent families has decreased. On the other hand, although single-parent households have a greater probability of experiencing labor income poverty, the difference with two-parent households has not varied throughout the study period.

\section{KEY WORDS}

Economic Crisis; Unemployment; Job Insecurity; Household 


\section{INTRODUCCIÓN}

En la última década, la precariedad laboral y el desempleo han aumentado de forma paralela a como lo ha hecho la diversidad de las estructuras familiares. La literatura sobre la sociología de la familia coincide en señalar que la estructura familiar se está convirtiendo en un mecanismo reproductor de la desigualdad como resultante del deterioro del mercado laboral, la fragilidad y la vulnerabilidad de las nuevas formas de familia (Boertien, Bernardi y Härkönen 2018; Cahn et al. 2018; Härkönen 2017; McLanahan y Percheski 2008). La Gran Recesión del año 2008 ha tenido un gran impacto en el mercado de trabajo de los países europeos (Van Gyes y Szekér 2013; International Labour Organization 2012). De estos países, el mercado laboral español ha sido de los más afectados, ya que, además de las consecuencias de la recesión global, ha sufrido el efecto de la propia crisis relacionada con la burbuja del sector de la construcción (Malo 2015; International Labour Organization 2011). Según los datos de la Encuesta de Población Activa realizada por el Instituto Nacional de Estadística (INE 2007; 2013), en España la tasa de desempleo pasó del $8.2 \%$ en 2007 al $26.1 \%$ en 2013. Además del constatado incremento del desempleo, en Europa se ha producido un aumento de determinadas formas de precariedad laboral, como el empleo a tiempo parcial o con bajo salario, siendo también España uno de los países más afectados por la expansión de este tipo de empleos (Vacas-Soriano 2018; Horemans, Marx y Nolan 2016; Ortiz García 2014).

La precarización laboral ha ido unida a la pluralización de las estructuras familiares y el deterioro económico de determinados hogares, como los monoparentales. Hecho que ha sido interpretado por los teóricos de la Segunda Transición Demográfica (STD) como el resultante del cambio cultural y demográfico asociado a estilos de vida más individualistas y desestandarizados, en el que el empoderamiento laboral y educativo de las mujeres ha tenido un papel decisivo en la toma de decisiones familiares como la fecundidad, el matrimonio o el divorcio (Lesthaeghe 2010; Mclanahan 2004). Los datos de la Encuesta Continua de Hogares (INE 2019) reflejan que en el año 2019 los hogares monoparentales representaban el $10 \%$ del total de hogares de España, estando la mayoría encabezados por mujeres $(81 \%)$. Pese a que existe una extensa literatura, tanto a nivel nacional como internacional, que ha demostrado el gran efecto de la crisis de 2008 en la situación laboral de la población en general y en el incremento de las desigualdades en los grupos más vulnerables, tales como las personas con un bajo nivel educativo (Rodríguez Hernández 2018; Sum y Khatiwada 2010), son menos los estudios que han analizado el caso concreto de los hogares con hijos según el tipo de familia, siendo en España muy limitados (Härkönen
2018; Nieuwenhuis y Maldonado 2018b). Entre esta escasa literatura podemos encontrar trabajos como el de Mattingly, Smith y Bean (2011) para Estados Unidos o el de Rafferty y Wiggan (2017) para Reino Unido, los cuales analizan la situación laboral de los padres según la estructura familiar. Destacan también los estudios, fundamentalmente en Estados Unidos, que han analizado la estructura familiar como vehículo reproductor de la desigualdad económica y como factor clave para garantizar el bienestar económico y emocional de los niños (Amato et al. 2015). Mclanahan (2004) habla de familias frágiles y de destinos familiares inciertos para referirse al deterioro económico de determinadas estructuras familiares más vulnerables, como las familias monoparentales o cohabitantes.

En general, los autores que analizan las consecuencias de la crisis encuentran que son los padres de las familias frágiles los más perjudicados por la peor situación del mercado de trabajo. No obstante, estos estudios, que toman como unidad de análisis la estructura familiar, focalizan su atención en el ámbito americano y anglosajón, y se centran casi exclusivamente en el impacto de la crisis en el corto plazo, siendo prácticamente inexistentes los estudios que abordan esta temática desde esa doble dimensión (familiar y longitudinal). El caso de España es de especial relevancia para estudiar las consecuencias de la crisis a largo plazo en los hogares con hijos, ya que es uno de los países desarrollados donde la recuperación del mercado de trabajo está siendo más lenta (OECD 2018).

El artículo contribuye de forma decisiva y novedosa al debate teórico y empírico sobre los efectos de la crisis y la posterior recuperación en la desigualdad laboral y económica según la estructura familiar en España, puesto que no hay muchos precedentes que hayan abordado esta temática desde el enfoque familiar longitudinal. La pregunta planteada hace referencia a cuál es la relación entre la estructura familiar, el desempleo y la precariedad laboral desde el inicio de la crisis económica hasta la etapa posterior de recuperación en España. Concretamente, el modelo analítico presentado examina la desigualdad y variabilidad existentes entre las familias monoparentales y biparentales en términos de intensidad laboral y bajos ingresos procedentes del trabajo en el periodo 2008-2017. Para conseguir tal fin, hemos utilizado los microdatos de la Encuesta de Condiciones de Vida del INE (2018).

Con este objetivo, el resto del artículo se estructura de la siguiente forma. Primero, se exponen los antecedentes teóricos sobre la aparición de nuevas formas familiares y su relación con la reproducción de la desigualdad. En segundo lugar, se presenta una revisión de la literatura sobre el impacto de la crisis en la situación laboral de los hogares según 
la estructura familiar, así como las hipótesis del estudio. Tercero, se especifican los detalles de la base de datos utilizada, las variables y la estrategia empírica. Posteriormente, se muestran los principales resultados de nuestros análisis. Por último, se resumen y se discuten las principales conclusiones del estudio.

\section{ESTRUCTURA FAMILIAR Y REPRODUCCIÓN DE LA DESIGUALDAD EN EL CONTEXTO DE LA SEGUNDA TRANSICIÓN DEMOGRÁFICA}

Una de las principales manifestaciones de la segunda transición demográfica (STD) es el cambio de valores y actitudes hacia estilos de vida más individualistas, acordes con los nuevos tipos de hogar que se crean más allá del modelo de varón sustentador, con un claro componente de género y resultantes de nuevas relaciones de pareja más igualitarias (Zaidi y Morgan 2017; Van De Kaa 1987). Estos procesos emergentes van acompañados de cambios en las formas familiares, que se hacen más frágiles e inestables (Goldscheider, Bernhardt y Lappegård 2015; Putnam 2015; McLanahan y Percheski 2008; Mclanahan 2004). La fragilidad de estas nuevas formas familiares se manifiesta en la proliferación de estructuras familiares vulnerables a los avatares del mercado laboral. De acuerdo con McLanahan y Percheski (2008), paralelamente al incremento de la diversidad familiar se ha producido un aumento de la desigualdad en los ingresos, convirtiéndose la estructura familiar en un mecanismo reproductor de la desigualdad debido a la distinta distribución de las estructuras familiares por educación, raza y género. En muchos casos, las estructuras familiares se convierten en un cúmulo de situaciones de desventaja económica. Es el caso de las familias monoparentales, en que las posiciones de oportunidad derivadas de factores como la educación inciden en las decisiones que se toman y en las oportunidades laborales.

El auge de estas nuevas formas de familias frágiles se ha interpretado desde el paradigma de la STD, el cual se centra en explicar cómo los cambios culturales dan razón de los cambios producidos en la fecundidad y las tipologías, como expresión del cambio de valores (Lesthaeghe 2010). De acuerdo con esta perspectiva interpretativa, los cambios en las estructuras familiares se consideran parte de un proceso de individualización y debilitamiento de los valores familiares tradicionales que organizaban la vida familiar, dando lugar a la diversidad y pluralidad de las familias. Las transiciones a estos nuevos estilos de vida tienen diferentes respuestas por parte de los individuos, en función de la trayectoria histórica y cultural de cada país, el contexto institucional y las circunstancias individuales de cada cual (Lesthaeghe 2010). Los cambios familiares asociados a la STD, traducidos en el incremento de nuevas formas de familia alternativas a la familia biparental tradicional, introducen nuevas formas de reproducción de la desigualdad relacionadas con la educación y la posición que ocupan los padres en el mercado laboral. La combinación de la precariedad y fragilidad de estas situaciones familiares se traslada tanto a la implicación de los padres en el cuidado de sus hijos como a la calidad de la misma dedicación, repercutiendo en el bienestar objetivo y subjetivo de los niños (Härkönen, Bernardi y Boertien 2017; Brown, Manning y Stykes 2015). Los teóricos de la STD han centrado su foco de atención en cómo el menor nivel educativo de los padres que pertenecen a familias frágiles repercute en la reproducción de la desigualdad y, por tanto, en la menor implicación de los padres, ya que tienen menos recursos y habilidades para afrontar las posibles desventajas, implicando esto un menor bienestar de los hijos (Härkönen 2018; Restrepo 2016; Main 2014; Rees, Goswami y Bradshaw 2010). Sin embargo, el interrogante de cómo la posición laboral de los padres asociada a la estructura familiar incide en la reproducción de las desigualdades en contextos de crisis económica ha sido menos estudiado. De ahí emana la pregunta que ha motivado este estudio de si la estructura familiar tiene alguna incidencia en la desigualdad laboral y económica en situaciones de crisis económica.

Los estudios disponibles han evidenciado que la precariedad e inestabilidad laborales que experimentan los padres que encabezan familias monoparentales, denominadas "familias frágiles", se asocia con déficits formativos que conducen a desempeñar trabajos mal pagados y escasamente cualificados, teniendo efectos negativos en el estrés laboral de los padres y, por tanto, en los indicadores de bienestar infantil subjetivos, tales como la satisfacción y las relaciones con amigos y la escuela de los niños (Hrženjak 2017; Flaquer, Moreno Mínguez y Cano López 2016; Heinrich 2014). Los resultados de estos estudios subrayan la necesidad de considerar la familia como una unidad de análisis, tanto en las dimensiones relativas a la estructura como a las características laborales y formativas de los padres, que inciden no solo en la situación económica de las familias, sino también en los estilos parentales $\mathrm{y}$, por lo tanto, en la educación y el futuro de sus hijos. A pesar del auge de los estudios sobre la diferente implicación de los padres según el tipo de familia, la situación laboral y el nivel educativo, así como los retos de la nueva paternidad (Flaquer, Moreno Mínguez y Cano López 2016; Lamb 1997), los estudios que relacionan estos términos con el bienestar infantil son más bien escasos (Wilson y Prior 2011).

En este trabajo analizaremos la asociación entre la estructura familiar, la intensidad en el trabajo 
y los ingresos laborales de los hogares, teniendo también en cuenta la incidencia de otros factores sociodemográficos, como el nivel educativo de los padres, con el fin último de motivar la relevancia de la estructura familiar como mecanismo reproductor de la desigualdad y de efectos adversos en el bienestar económico y emocional de los niños (Härkönen 2017; Mclanahan 2004; Amato y Fowler 2002). La lógica interpretativa de este argumento propio de la STD es que los individuos con menor formación acceden a peores trabajos, experimentan en mayor medida el desempleo y están también proporcionalmente más afectados por el divorcio, la monoparentalidad y la cohabitación (las llamadas familias frágiles), lo que, unido a que tienen más hijos y a edades más tempranas en posiciones socio-económicas desventajosas, conduce a una peor situación de sus hijos tanto en términos de bienestar objetivo como subjetivo (McLanahan y Percheski 2008; Härkönen y Dronkers 2006), contribuyendo esto a la acumulación y reproducción de las desventajas económicas y culturales en estos niños a lo largo de su vida (Dannefer 2003). Las encuestas, datos y estudios disponibles nos permiten aproximaciones muy limitadas a esta contrastación empírica, pero sí nos permiten elaborar hipótesis para plantear interrogantes que abren nuevos caminos de investigación.

Si a esto unimos que la crisis económica ha creado un nuevo escenario laboral más precario e inestable, empeorando la situación socioeconómica de las familias, fundamentalmente de las más frágiles o en desventaja económica (Nieuwenhuis y Maldonado 2018b; Garfinkel, McLanahan y Wimer 2016), es de esperar que este contexto afecte a las oportunidades, satisfacción y comportamientos de los hijos $y$, por tanto, a la reproducción intergeneracional de la desigualdad (Cohen 2015; Brand y Simon Thomas 2014). Sin embargo, los déficits educativos, cognitivos y relacionales de los hijos heredados de sus familias de origen podrían corregirse con una política social adecuada (Boertien, Bernardi y Härkönen 2018; Bernardi y Radl 2014; Pong, Dronkers y Hampden-Thompson 2003). En definitiva, los resultados de los estudios que incorporan la estructura familiar son de suma importancia para diseñar políticas familiares que contrarresten los efectos negativos de las estructuras familiares en el bienestar de los niños. A este respecto, contamos con numerosos estudios en la literatura comparada sobre los estados de bienestar que centran la atención en analizar la importancia de las políticas públicas para mitigar los efectos negativos de los factores socioeconómicos y culturales que median en la reproducción de la desigualdad social (Maldonado y Nieuwenhuis 2015; Brady y Burroway 2012; Pong, Dronkers y Hampden-Thompson 2003). Estas evidencias empíricas disponibles constatan la necesi- dad de analizar los factores familiares y laborales que contribuyen a la reproducción intergeneracional de la desigualdad. Solo con un diagnóstico previo podremos activar las políticas sociales adecuadas a cada situación y contexto regional y cultural.

\section{LA ESTRUCTURA FAMILIAR COMO FACTOR DETERMINANTE DE LA SITUACIÓN LABORAL EN EL PERIODO DE CRISIS ECONÓMICA}

Una amplia literatura ha examinado las distintas consecuencias del deterioro laboral en los individuos según ciertos factores sociodemográficos tales como el nivel educativo, la edad o la nacionalidad (Rodríguez Hernández 2018; Horemans, Marx y Nolan 2016; Mooi-Reci y Muñoz-Comet 2016; Aysa-Lastra y Cachón 2015; Miguélez y López-Roldán 2014; Bell y Blanchflower 2011; Sum y Khatiwada 2010; Verick 2009). En general, en todos estos trabajos se muestra que el incremento del desempleo y de la precariedad laboral, a raíz de la crisis económica del 2008, afectó especialmente a los colectivos peor posicionados según las características expuestas.

Son limitados los estudios que han focalizado la atención en la situación laboral específica de los padres. En concreto, estos se centran en comparar a los padres de familias monoparentales y biparentales. Entre estas escasas contribuciones se encuentra el trabajo de Mattingly, Smith y Bean (2011), en el que se muestra que, para el caso de Estados Unidos, entre 2007 y 2010 el porcentaje de padres monoparentales en desempleo aumentó más que el de padres casados. De hecho, los datos reportados por estos autores indican que ya en 2007 la tasa de desempleo de los padres que encabezan hogares monoparentales era superior a la de los casados, por lo que esta diferencia aumentó durante los años de recesión. Rafferty y Wiggan (2017) han estudiado la evolución de la situación laboral de las madres monoparentales y biparentales en el periodo 2005-2013 en el Reino Unido. Entre sus principales resultados, destaca el hecho de que las madres monoparentales tienen una mayor probabilidad de experimentar situaciones de desempleo, empleo a tiempo parcial involuntario y subempleo, y que estas diferencias con las madres biparentales se han ampliado desde el inicio de la crisis. Aunque no es el principal objetivo de su trabajo, Treanor (2018) analiza para Escocia la evolución de la intensidad laboral en los hogares biparentales y monoparentales entre 2008 y 2012. Los resultados indican que los hogares monoparentales presentan una menor intensidad laboral que los biparentales y que ambos hogares disminuyeron su intensidad en el empleo entre 2008 y 2009. No obstante, en el periodo 2010-2012 los biparentales se recuperaron y los monoparentales 
continuaron experimentando un decrecimiento de la intensidad laboral.

En general, en todos estos trabajos se observa la mayor precariedad e inestabilidad laboral de las familias monoparentales en la etapa de crisis. Gran parte de las desventajas y la precariedad laboral que experimentan estas familias puede explicarse por la desigualdad de género existente en el mercado de trabajo, ya que la mayoría de los hogares monoparentales están encabezados por mujeres (Nieuwenhuis y Maldonado 2018a; 2018b). Los estudios realizados en Europa han evidenciado que los hogares monoparentales en los que la madre está desempleada y tiene una reducida cualificación se convierten en hogares con elevado riesgo de pobreza infantil si no hay una política social y laboral orientada a favorecer la inserción laboral estable y digna de esas madres (Madruga Torremocha 2006; Rodríguez Sumaza 2001). Por lo tanto, los estudios disponibles para las familias monoparentales evidencian que los ingresos procedentes del trabajo, la educación de los progenitores y las prácticas de crianza, además de ser claves para el bienestar de los niños, son una problemática asociada al género que reproduce y explica la desigualdad observada cuando se introduce la variable del hogar (Kollmeyer 2013; Heckman 2006; Amato y Fowler 2002). De ahí la gran relevancia de los resultados que presentamos en este artículo, ya que numerosos estudios han evidenciado que los niños que crecen en familias en desventaja tendrán más dificultades y menos oportunidades para hacer frente a los retos del futuro, por lo que la familia se convierte así en una estructura reproductora de la desigualdad estratificada por género y procedencia étnica (Erman y Härkönen 2017; Duncan, Ziol-Guest y Kalil 2010; Heckman 2006).

A esto se une que los padres y madres que encabezan los hogares monoparentales, al ser los únicos proveedores de ingresos de su hogar, tienen menos recursos para conciliar vida laboral y familiar que los padres biparentales, lo cual repercute en su menor oportunidad para poder formarse, promocionarse y competir por empleos de calidad. Por tanto, estas situaciones redundan generalmente en que los progenitores de hogares monoparentales, generalmente mujeres, terminan desempeñando empleos más precarios e inestables (Esser y Olsen 2018). Si bien las escasas evidencias empíricas disponibles se han limitado a cuantificar la variación en las estructuras familiares según el gradiente educativo, cada vez es mayor el interés por estudiar cómo las diferencias socioeconómicas referidas a otras variables, como la situación laboral o el tipo de empleo, son amplificadas según el tipo de estructura familiar (Boertien, Bernardi y Härkönen 2018; Matysiak, Styrc y Vignoli 2014).

\section{HIPÓTESIS}

En general, los estudios previos han mostrado que, antes del inicio de la recesión de 2008, los hogares monoparentales, en comparación con los biparentales, se enfrentaban a una peor situación en el mercado laboral, la cual se acentuó tras la crisis económica. Esta diferencia se mantiene incluso teniendo en cuenta otras características de las familias, tales como el nivel educativo o la edad de los padres (Rafferty y Wiggan 2017; Mattingly, Smith y Bean 2011). Dada la lenta recuperación del mercado de trabajo español una vez finalizado el periodo de recesión (OECD 2018), en este trabajo se plantean dos hipótesis. En primer lugar, hay que considerar que, como señala Treanor (2018), la gran incidencia del incremento del desempleo y del empleo a tiempo parcial en las familias monoparentales evidencia una reducción de la intensidad laboral de estos hogares. Concretamente, la medida de intensidad en el empleo que utilizan estos autores introduce la variable relativa a si los padres del hogar trabajan o no y, en el que caso de que trabajen, el tipo de jornada laboral (tiempo completo o tiempo parcial). Por tanto, la primera hipótesis de este trabajo postula que, tanto al final del periodo de recesión como en la etapa posterior de recuperación, la diferencia entre los hogares monoparentales y biparentales en términos de intensidad laboral será superior a la del inicio de la crisis.

Por otra parte, también hay que tener en cuenta que el aumento del desempleo y de determinados tipos de precariedad laboral, como el empleo a tiempo parcial y los bajos salarios, repercute en la disminución de los ingresos procedentes del trabajo. Por consiguiente, nuestra segunda hipótesis apunta a que la diferencia en la probabilidad de tener bajos ingresos laborales entre las familias monoparentales y biparentales será mayor al final del periodo de recesión y en los años posteriores de recuperación que al comienzo de la crisis. La motivación de esta segunda hipótesis se encuentra en los estudios realizados para Estados Unidos y Europa, donde se ha observado que la crisis ha amplificado las diferencias según el tipo de familia, contribuyendo al empeoramiento de la situación laboral y económica de las familias monoparentales encabezadas por mujeres (Nieuwenhuis y Maldonado 2018b; Cantillon et al. 2017), con las repercusiones negativas que esto tiene para el bienestar y la pobreza de la infancia.

\section{DATOS, VARIABLES Y MÉTODO}

En este artículo se utilizan los datos de la Encuesta de Condiciones de Vida (ECV) del INE (2018). Esta base de datos proporciona una amplia información de los hogares y de ciertas ca- 
racterísticas de cada uno de sus miembros como la edad, el nivel educativo, la nacionalidad, la situación laboral y los ingresos que obtienen por su trabajo. Para los análisis usamos los ficheros de datos transversales de los años 2008, 2013 y 2017, lo que nos va a permitir estudiar la situación de las familias desde el inicio de la recesión hasta el periodo de recuperación económica ${ }^{1}$. Nuestra población objeto de estudio se compone de todos aquellos hogares en los que residen padres con hijos menores de 18 años.

La primera de nuestras variables dependientes mide la intensidad laboral del hogar. Utilizamos de referencia la variable de Treanor (2018), la cual toma el valor 1 si todos los padres del hogar ${ }^{2}$ trabajan a tiempo completo; 0.75 si uno de ellos trabaja a tiempo completo y el otro a tiempo parcial; 0.5 si todos los padres del hogar trabajan a tiempo parcial o uno de los padres trabaja a tiempo completo y el otro no trabaja; 0.25 si uno de los padres trabaja a tiempo parcial y el otro no trabaja, y 0 si ninguno de los padres trabaja ${ }^{3}$. En nuestro caso, para identificar los hogares con una intensidad laboral media o baja, creamos una variable binaria cuyo valor 1 se corresponde con los valores $0,0.25$ y 0.5 de la variable anterior, mientras que para los valores 1 y 0.75 toma el valor 0 .

En lo que respecta a la segunda variable dependiente, siguiendo a Nieuwenhuis y Maldonado (2018a), esta identifica la pobreza de ingresos laborales. Concretamente, examinamos la pobreza de ingresos laborales brutos, ya que estos no se ven afectados por la fiscalidad directa ${ }^{4}$. La variable toma el valor $1 \mathrm{si}$ los ingresos laborales del hogar son inferiores al $60 \%$ de la mediana nacional y 0 en caso contrario ${ }^{5}$. Este umbral es el mismo que el utilizado por la medida estándar de pobreza de ingresos de la Unión Europea (lacovou 2013). Además, ajustamos los ingresos por inflación y usamos el mismo límite de bajos ingresos del año 2008 para los años posteriores. Al anclar el umbral de pobreza, este no varía según los cambios en la mediana de los ingresos laborales a lo largo del periodo de estudio, lo cual permite examinar con mayor exactitud el impacto de la crisis en las familias (Martínez y Navarro 2014) ${ }^{6}$.

En cuanto a las variables independientes, la principal variable de estructura familiar indica si el hogar es monoparental o biparental. A este respecto, aunque la $\mathrm{ECV}$ ofrece una variable que identifica distintos tipos de hogares con hijos, Chzhen y Bradshaw (2012) señalan que esta no clasifica como hogares monoparentales a aquellos en los que convive una unidad familiar monoparental con otros adultos, tales como los abuelos de los niños. Por tanto, de manera similar a estos autores, también definimos estos hogares como monoparentales ${ }^{7}$. Del mismo modo, clasificamos como hogares biparentales todos aquellos en los que vive una pareja de padres con sus hijos ${ }^{8}$, independientemente de que haya más adultos en el hogar.

Por otro lado, como variables independientes de control se incluyen características sociodemográficas de las familias que también se han utilizado en estudios previos (Chzhen 2017; Rafferty y Wiggan 2017; Mattingly, Smith y Bean 2011). En primer lugar, se tiene en cuenta el nivel educativo alcanzado por el padre o madre del hogar con mayor nivel, el cual puede ser: nivel alto (educación terciaria), nivel medio (educación secundaria o postsecundaria no terciaria) o nivel bajo (primera etapa de educación secundaria, primaria, pre-primaria o ningún tipo de educación). Asimismo, se incluye una variable que señala si el padre o madre con más edad del hogar tiene más de 30 años o si, por el contrario, tiene 30 años o menos. Se distinguen tres tipos de hogares según la nacionalidad de los padres: hogares donde al menos uno de los padres tiene nacionalidad española, hogares de padres extranjeros en los que al menos uno de los padres tiene nacionalidad de un país europeo distinto a España, y hogares en los que todos los padres tienen nacionalidad de un país no europeo. También se tiene en cuenta el número de niños en el hogar, diferenciando entre aquellos hogares con uno, dos o tres o más niños. Por último, se incluye un variable que indica si el niño más pequeño del hogar es menor de 12 años o tiene entre 12 y 17 años.

El análisis empírico de este trabajo consta de dos partes. Primero, se realiza un análisis descriptivo de las características sociodemográficas de las familias monoparentales y biparentales. Asimismo, se muestran los porcentajes de familias monoparentales y biparentales con intensidad laboral media o baja y con bajos ingresos en los años 2008, 2013 y 2017. Tras esta primera exploración, agrupando los datos transversales de los tres años de estudio, se estiman dos tipos de modelos de regresión logística para cada una de las variables dependientes. El primer modelo analiza la relación entre la estructura familiar y las variables de intensidad laboral y bajos ingresos laborales, incluyendo en la estimación el resto de las variables sociodemográficas de control y dummies anuales. Posteriormente, para examinar la evolución de esta relación a lo largo del periodo de estudio y poder contrastar nuestras hipótesis, se estima un segundo tipo de modelo similar al anterior en el que se incluye la interacción entre la variable de estructura familiar y las dummies anuales. 


\section{RESULTADOS}

\section{Análisis descriptivo}

En la tabla 1 se presentan las características sociodemográficas de los hogares según la estructura familiar. De la información mostrada, destacan especialmente las grandes diferencias entre los dos tipos de hogares en lo que respecta al nivel educativo de los padres. En el $46 \%$ de los hogares monoparentales los padres tienen un nivel educativo bajo, mientras que en los hogares biparentales el porcentaje es del $27 \%$. Por el contrario, en casi la mitad de los hogares biparentales al menos uno de los padres tiene un nivel educativo alto, frente al $27 \%$ de los monoparentales. Por otra parte, la presencia de padres de 30 años o menos es superior en los hogares monoparentales. En cuanto a la nacionalidad de los padres, no hay grandes diferencias entre los dos tipos de hogares, aunque es algo mayor el porcentaje de hogares monoparentales con padres procedentes de países no europeos ( $12 \%$ frente al $8 \%$ de los hogares biparentales). Los datos también reportan que, en general, en los hogares monoparentales hay menos niños que en los biparentales. Por último, el porcentaje de hogares en los que el hijo más peque-

Tabla 1.

Características sociodemográficas de los hogares según estructura familiar

\begin{tabular}{|c|c|c|}
\hline & $\begin{array}{c}\text { Hogares } \\
\text { monoparentales }\end{array}$ & $\begin{array}{c}\text { Hogares } \\
\text { biparentales }\end{array}$ \\
\hline \multicolumn{3}{|c|}{ Nivel educativo de los padres (\%) } \\
\hline Nivel alto & 26.54 & 48.43 \\
\hline Nivel medio & 27.07 & 24.77 \\
\hline Nivel bajo & 46.39 & 26.80 \\
\hline \multicolumn{3}{|l|}{ Edad de los padres (\%) } \\
\hline Más de 30 años & 82.97 & 96.33 \\
\hline 30 años o menos & 17.03 & 3.67 \\
\hline \multicolumn{3}{|c|}{ País de origen de los padres (\%) } \\
\hline España & 85.62 & 87.79 \\
\hline Resto de la UE & 2.53 & 3.74 \\
\hline País no miembro UE & 11.85 & 8.47 \\
\hline \multicolumn{3}{|l|}{ Número de hijos (\%) } \\
\hline Uno & 74.99 & 53.12 \\
\hline Dos & 20.25 & 39.36 \\
\hline Tres o más & 4.76 & 7.52 \\
\hline \multicolumn{3}{|l|}{ Edad del hijo menor (\%) } \\
\hline $12-17$ & 35.28 & 25.88 \\
\hline $0-11$ & 64.72 & 74.12 \\
\hline Muestra & 1,611 & 9,957 \\
\hline
\end{tabular}

ño tiene menos de 12 años es algo mayor en los biparentales $(74 \%)$ que en los monoparentales $(65 \%)$.

La tabla 2 muestra, en cada uno de los años de estudio, los porcentajes de hogares monoparentales y biparentales con una intensidad laboral media o baja. Los datos indican que en el año 2008 el porcentaje de los hogares biparentales (45\%) era superior al de los hogares monoparentales (39\%). A este respecto, hay que señalar que, según nuestra muestra, el porcentaje de familias biparentales en las que los dos padres trabajan es inferior al $60 \%$ en los tres años de estudio (véase la tabla A1 del anexo), el cual es algo menor al de la mayoría de los países desarrollados (Thévenon 2011). No obstante, tras la crisis económica la diferencia entre los hogares monoparentales y biparentales se ha invertido. Esto se debe a que, si bien en el periodo 2008-2013 hubo un incremento de hogares con intensidad laboral media o baja para ambos tipos de familias, este fue mayor en las monoparentales, las cuales, a su vez, tuvieron una menor disminución en el periodo posterior (2013-2017). De hecho, si bien en 2017 las familias biparentales presentaban un menor porcentaje que en 2008 , las monoparentales todavía no habían recuperado el nivel previo a la crisis.

Tabla 2.

Porcentaje de hogares monoparentales y biparentales con intensidad laboral media o baja, 2008- 2017

\begin{tabular}{|l|c|c|c|}
\hline & $\mathbf{2 0 0 8}$ & $\mathbf{2 0 1 3}$ & $\mathbf{2 0 1 7}$ \\
\hline Hogares monoparentales & 39.38 & 56.37 & 47.02 \\
\hline Hogares biparentales & 45.34 & 56.55 & 40.64 \\
\hline
\end{tabular}

Fuente: Elaboración propia a partir de las ECV de 2008, 2013 y 2017

Los datos de la tabla A2 del anexo revelan que el mayor incremento de hogares con baja intensidad laboral en los monoparentales está explicado, en gran parte, por la mayor pérdida de empleo por parte de los padres de este tipo de familias, en comparación con los padres biparentales. Además, también hay que tener en cuenta el incremento de la incidencia del trabajo a tiempo parcial en los hogares monoparentales durante la etapa de crisis económica.

En la tabla 3 se presenta el porcentaje de hogares en situación de pobreza por bajos ingresos laborales. Los datos muestran que existe una gran diferencia entre las familias monoparentales y biparentales. Concretamente, en el año 2008 el 46 $\%$ de hogares monoparentales tenían bajos ingresos laborales, frente al $20 \%$ de los biparentales. En 2013 ambos porcentajes aumentaron, sin que la distancia entre ellos variase de manera notable. No obstante, entre 2013 y 2017 , los hogares biparentales experimentaron un leve descenso, mientras que 
los monoparentales se mantuvieron estables. En consecuencia, en 2017 la incidencia de la pobreza de ingresos laborales era todavía superior a la del 2008 en ambos tipos de hogares, especialmente en los monoparentales.

Tabla 3.

Porcentaje de hogares monoparentales y biparentales en situación de pobreza por bajos ingresos laborales, 2008- 2017

\begin{tabular}{|l|l|l|l|}
\hline & $\mathbf{2 0 0 8}$ & $\mathbf{2 0 1 3}$ & $\mathbf{2 0 1 7}$ \\
\hline Hogares monoparentales & 45.91 & 57.08 & 59.66 \\
\hline Hogares biparentales & 19.92 & 32.54 & 26.55 \\
\hline
\end{tabular}

Fuente: Elaboración propia a partir de las ECV de 2008, 2013 y 2017

\section{Modelos multivariantes}

A continuación, se presentan los resultados de los modelos de regresión logística. La primera parte de estos resultados se muestra en la tabla 4 y corresponde a la variable dependiente de intensidad laboral. De acuerdo con los resultados del modelo 1 , tras controlar por el resto de las características sociodemográficas, las familias monoparentales presentan una menor probabilidad de tener una intensidad laboral media o baja que las familias biparentales. En lo que respecta a los resultados de las variables de control, en primer lugar, se puede apreciar que los hogares de padres con nivel educativo medio y nivel educativo bajo registran una mayor probabilidad de tener una menor intensidad laboral que los hogares de padres con un nivel alto. La edad también se muestra como un factor determinante, ya que para las familias cuyos padres tienen 30 años o menos la probabilidad de experimentar situaciones de intensidad laboral media o baja es mayor que para las familias de padres de más de 30 años. En lo referente a la nacionalidad, tanto los hogares de padres inmigrantes de países de la UE como de países no europeos presentan una mayor probabilidad de tener una intensidad laboral media o baja. Por otro lado, en los hogares con tres niños o más hay un mayor riesgo de afrontar situaciones de menor intensidad laboral que en aquellos en los que solo hay un niño. Sin embargo, no hay diferencias significativas entre los hogares de uno y dos niños. En cuanto a la variable de edad del niño más pequeño, su coeficiente no es estadísticamente significativo. Por último, los resultados de las variables anuales señalan que, en comparación con el año 2008, el riesgo de que todos los hogares experimente una menor intensidad laboral es mayor en 2013, no observándose este resultado en 2017.
Tabla 4.

Regresión logística sobre la probabilidad de tener intensidad laboral media o baja

\begin{tabular}{|c|c|c|}
\hline & Modelo 1 & Modelo 2 \\
\hline \multicolumn{3}{|c|}{ Estructura familiar (ref.: biparental) } \\
\hline Monoparental & $-0.370^{* * *}$ & $-0.684^{* * *}$ \\
\hline \multicolumn{3}{|c|}{ Nivel educativo de los padres (ref.: nivel alto) } \\
\hline Nivel medio & $0.716^{\star \star *}$ & $0.716^{\star * \star}$ \\
\hline Nivel bajo & $1.399^{* * *}$ & $1.397^{* * *}$ \\
\hline \multicolumn{3}{|c|}{ Edad de los padres (ref.: más de 30 años) } \\
\hline 30 años o menos & $0.621^{* * *}$ & $0.626^{* * *}$ \\
\hline \multicolumn{3}{|c|}{ País de origen de los padres (ref.: España) } \\
\hline Resto de la UE & $0.452^{* *}$ & $0.446^{* *}$ \\
\hline País no miembro UE & $0.566^{* * *}$ & $0.574^{* * *}$ \\
\hline \multicolumn{3}{|c|}{ Número de hijos (ref.: uno) } \\
\hline Dos & 0.028 & 0.028 \\
\hline Tres o más & $0.460^{* * *}$ & $0.460^{\star * *}$ \\
\hline \multicolumn{3}{|c|}{ Edad del hijo menor (ref.: 12-17) } \\
\hline $0-11$ & -0.054 & -0.051 \\
\hline \multicolumn{3}{|l|}{ Años (ref.:2008) } \\
\hline 2013 & $0.643^{* * *}$ & $0.611^{* * *}$ \\
\hline 2017 & 0.079 & 0.020 \\
\hline \multicolumn{3}{|l|}{ Estructura familiar*Años } \\
\hline Monoparental*2013 & & $0.332^{*}$ \\
\hline Monoparental`2017 & & $0.515^{\star * *}$ \\
\hline Pseudo $\mathrm{R}^{2}$ & 0.087 & 0.088 \\
\hline Observaciones & 11,559 & 11,559 \\
\hline
\end{tabular}

${ }^{* * *} p<0.01,{ }^{* *} p<0.05,{ }^{*} p<0.1$

Fuente: Elaboración propia a partir de las ECV de 2008, 2013 y 2017

En el modelo 2 se incluye en la estimación las interacciones entre la variable de familia monoparental y las dummies anuales de 2013 y 2017. Los coeficientes de ambas interacciones son positivos y significativos. Para facilitar su interpretación, los resultados de estas interacciones se presentan gráficamente a través de las probabilidades predichas. En la figura 1 se aprecia que, si bien la probabilidad de que los hogares monoparentales tenga una baja intensidad laboral es menor que en los hogares biparentales, la distancia entre los dos grupos de familias disminuyó tanto en 2013 como en 2017. Esto se debe, en primer lugar, a que las probabilidades de ambos hogares aumentaron en 2013, siendo este incremento mayor en los monoparentales. Además, en 2017 la probabilidad de los hogares monoparentales disminuyó menos que la de los biparentales. Por tanto, aunque no podemos corroborar totalmente nuestra primera hipótesis, ya que las familias monoparentales parten de una mejor situación en términos de intensidad de trabajo, sí se puede observar que la disminución de la intensidad laboral, como consecuencia de la crisis, ha afectado en mayor medida a este tipo de familias. 
Figura 1.

Probabilidades predichas para la interacción entre el tipo de hogar y el año de la encuesta. Intensidad laboral media o baja.

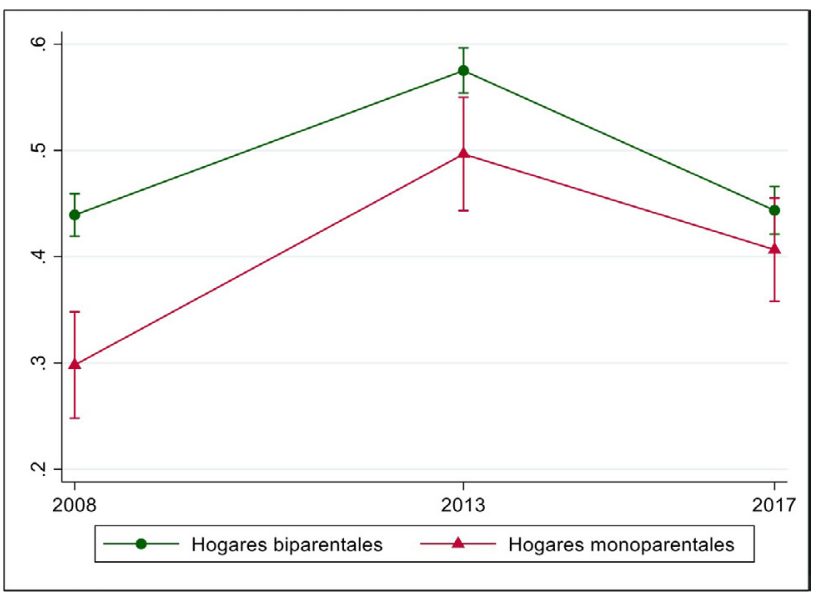

Nota: Probabilidades calculadas a partir del modelo 2 de la tabla 4

Tabla 5.

Regresión logística sobre la probabilidad de tener bajos ingresos laborales

\begin{tabular}{|c|c|c|}
\hline & Modelo 1 & Modelo 2 \\
\hline \multicolumn{3}{|c|}{ Estructura familiar (ref.: biparental) } \\
\hline Monoparental & $1.037^{\star * *}$ & $1.035^{\star * *}$ \\
\hline \multicolumn{3}{|c|}{ Nivel educativo de los padres (ref.: nivel alto) } \\
\hline Nivel medio & $1.047^{* * *}$ & $1.046^{* * *}$ \\
\hline Nivel bajo & $1.851^{* * *}$ & $1.850^{\star * *}$ \\
\hline \multicolumn{3}{|c|}{ Edad de los padres (ref.: más de 30 años) } \\
\hline 30 años o menos & 0.228 & 0.231 \\
\hline \multicolumn{3}{|c|}{ País de origen de los padres (ref.: España) } \\
\hline Resto de la UE & $1.296^{* * *}$ & $1.292^{* * *}$ \\
\hline País no miembro UE & $1.055^{\star * *}$ & $1.053^{* * *}$ \\
\hline \multicolumn{3}{|c|}{ Número de hijos (ref.: uno) } \\
\hline Dos & $0.228^{* * *}$ & $0.228^{\star * *}$ \\
\hline Tres o más & $0.970^{* * *}$ & $0.971^{* * *}$ \\
\hline \multicolumn{3}{|c|}{ Edad del hijo menor (ref.: 12-17) } \\
\hline $0-11$ & 0.109 & 0.109 \\
\hline \multicolumn{3}{|l|}{ Años (ref.:2008) } \\
\hline 2013 & $0.899^{* * *}$ & $0.919^{\star * *}$ \\
\hline 2017 & $0.786^{\star * *}$ & $0.760^{* * *}$ \\
\hline \multicolumn{3}{|l|}{ Estructura familiar*Años } \\
\hline Monoparental²013 & & -0.146 \\
\hline Monoparental`2017 & & 0.145 \\
\hline Pseudo $\mathrm{R}^{2}$ & 0.176 & 0.176 \\
\hline Observaciones & 11,568 & 11,568 \\
\hline
\end{tabular}

${ }^{* * *} p<0.01,{ }^{* *} p<0.05,{ }^{*} p<0.1$

Fuente: Elaboración propia a partir de las ECV de 2008, 2013 y 2017
En la tabla 5 se muestran los resultados para la variable de bajos ingresos por trabajo. En el modelo 1 se observa que los hogares monoparentales registran una mayor probabilidad de experimentar pobreza por bajos ingresos laborales que los hogares biparentales, resultado que es acorde con los hallazgos descriptivos mostrados en la tabla 3 . En cuanto a las variables de control, los resultados señalan la mayor probabilidad de tener bajos ingresos por parte de los hogares de padres con nivel educativo medio y bajo o inmigrantes, en comparación con sus respectivas categorías de referencia. No hay diferencias significativas entre los hogares según la edad de los padres. Por otra parte, aquellas familias con más de un niño registran una mayor probabilidad de tener bajos ingresos. La edad del niño más pequeño parece no tener ninguna repercusión. Finalmente, los resultados indican que, tanto en el año 2013 como en el 2017 la probabilidad de que todos los hogares tengan bajos ingresos procedentes del trabajo es mayor que en el año 2008.

En el segundo modelo, ninguno de los coeficientes de las variables de interacción es estadísticamente significativo, por lo que se puede deducir que, en lo que respecta a la probabilidad de tener bajos ingresos, la diferencia entre las familias monoparentales y biparentales no ha variado desde el inicio de la crisis económica. En consecuencia, estos resultados no nos permiten confirmar la segunda hipótesis en ninguna de las dos etapas de estudio.

\section{DISCUSIÓN Y CONCLUSIONES}

El principal objetivo de este trabajo ha sido estudiar la desigualdad entre familias monoparentales y biparentales en términos de intensidad en el empleo y bajos ingresos laborales en el periodo 2008-2017. En primer lugar, en lo que respecta a la intensidad laboral de los hogares, los resultados sostienen que desde el año 2008 ha disminuido la ventaja que presentan las familias monoparentales con respecto a las biparentales. Esto refleja el mayor impacto del aumento del empleo a tiempo parcial y, sobre todo, del desempleo en las familias monoparentales, lo que está en línea con investigaciones anteriores (Rafferty y Wiggan 2017; Mattingly, Smith y Bean 2011). Sin embargo, que este tipo de hogares presenten una menor probabilidad de tener una intensidad laboral media o baja es un resultado contrario a lo que cabía esperar según la literatura disponible. Esto puede deberse a que España es uno de los países europeos donde se observa con mayor frecuencia el modelo familiar biparental de un solo sustentador económico (Moreno Mínguez y Crespi 2017). Por otro lado, también hay que señalar que la recuperación del mercado de trabajo en el periodo posterior a la crisis parece estar beneficiando principalmente a las familias biparentales (Treanor 2018). 
En segundo lugar, los resultados también muestran que las familias monoparentales presentan una mayor probabilidad de experimentar bajos ingresos laborales y desventaja económica que las biparentales, lo cual es debido fundamentalmente a que se trata de familias encabezadas mayoritariamente por mujeres que acusan mayor precariedad e inestabilidad laboral (Härkönen 2018; Nieuwenhuis y Maldonado 2018a; McLanahan y Percheski 2008). En la misma línea de análisis, los estudios comparados han corroborado esta idea al constatar que la probabilidad de pobreza es mayor entre los hogares monoparentales (Härkönen 2017). No obstante, a pesar del crecimiento de la incidencia de la pobreza por bajos ingresos laborales desde el inicio de la crisis de 2008, los resultados apuntan a que no parecen haber aumentado significativamente las diferencias según la estructura familiar en España. Estos resultados, en parte, no concuerdan con los presentados a nivel internacional, en los que se ha evidenciado que la estructura familiar incide claramente en la amplificación de la desigualdad de ingresos en Estados Unidos y Europa (Alamillo 2016; Kollmeyer 2013; Esping-Andersen 2007). En este sentido, podemos observar que la mayor disminución de la intensidad laboral en los hogares monoparentales no ha implicado un mayor aumento en la probabilidad de tener bajos ingresos laborales, con respecto a los biparentales. Esto puede deberse a que, aunque el aumento del empleo a tiempo parcial en el periodo de crisis afectase en mayor medida a los hogares monoparentales, el nivel de ingresos que se obtiene en este tipo de empleos puede ser superior al umbral de pobreza si trabajan un número suficiente de horas (Salladarré y Hlaimi 2014; Baxter et al. 2012). Además, cabe añadir que, si bien el aumento del desempleo afectó severamente a las familias monoparentales, este ha podido ser un desempleo de corta duración, ya que es muy probable que la precariedad económica fuera más intensa en estos hogares debido a la existencia de un solo proveedor de ingresos, lo que activó la búsqueda de empleo para salir lo antes posible de dicha situación (Eamon y Wu 2013).

En definitiva, los resultados obtenidos han constatado que las negativas consecuencias de la crisis económica en la intensidad laboral han perjudicado especialmente a los hogares monoparentales, y que el efecto de la estructura familiar en la probabilidad de experimentar bajos ingresos laborales ha permanecido relativamente estable desde 2008 a 2017. En cualquier caso, los hallazgos muestran la desventaja económica de las familias monoparentales (Nieuwenhuis y Maldonado 2018b; Cantillon et al. 2017). Por tanto, la estructura familiar tiene un papel fundamental en la reproducción intergeneracional de la desigualdad en España, ya que tanto la mayor vulnerabilidad de los hogares monoparentales a la disminución de la intensidad en el empleo como la peor posición económica de estas familias a lo largo de todo el periodo de estudio se trasladan al bienestar infantil y a las oportunidades futuras de los niños de estos hogares (Cohen 2015; Brand y Simon Thomas 2014). La estructura familiar es, por lo tanto, un factor vehicular fundamental para explicar la desigualdad económica, la desigualdad laboral, la pobreza infantil, el comportamiento, enfermedades y rendimiento de los niños desfavorecidos que viven en determinados tipos de familia.

Los resultados de nuestro estudio también han constatado la relevancia de otros factores sociodemográficos. En particular, aquellos hogares con un mayor número de niños y de padres con un bajo nivel educativo, jóvenes o inmigrantes, tienen una mayor probabilidad de experimentar baja intensidad laboral y reducidos ingresos. En general, estos resultados están en la línea con las investigaciones previas realizadas en los países del entorno europeo y Estados Unidos (Chzhen 2017; Mooi-Reci y Muñoz-Comet 2016; Bell y Blanchflower 2011; Sum y Khatiwada 2010; Verick 2009). Este hallazgo tiene gran relevancia empírica y de transferencia para el diseño de políticas públicas, ya que demuestra que, para España, la desventaja asociada a factores como el nivel educativo o la edad, además de reducir oportunidades de índole personal, también tiene efectos negativos en los hogares vulnerables, lo que repercute en los estilos parentales y en el bienestar infantil.

Los hallazgos presentados en este artículo nos permiten concluir que, diez años después del inicio de la crisis económica, nos encontramos ante un escenario laboral más precario e inestable, el cual está perjudicando la recuperación de las familias, fundamentalmente de las más desfavorecidas y frágiles (monoparentales). A la luz de estos resultados, parece prioritario introducir la variable familiar en el diseño de las políticas públicas destinadas a mitigar las consecuencias del aumento de la precariedad, yendo más allá de las reformas laborales, ya que estas políticas no solo deberían centrarse en reducir el desempleo, sino también en la disminución de la inestabilidad y la precariedad laborales que afectan a las familias más vulnerables y que pueden contribuir a la reproducción intergeneracional de la desigualdad.

Aunque este tipo de estudios se han multiplicado en los últimos años a nivel internacional, se requieren más análisis empíricos como el presente, que avalen el argumento de que la variación en la estructura familiar es un factor importante para explicar la reproducción de la desigualdad socioeconómica de las oportunidades, puesto que aún no contamos con suficiente respaldo empírico, siendo este especialmente inexistente para el caso español.

Sirvan los resultados de este estudio para contribuir al debate sobre la necesidad de realizar más investigaciones que presenten mayor atención a la situación laboral y económica de las familias con hijos, 
así como a las consecuencias que está teniendo la precariedad resultante de la crisis de 2008. Esta reflexión es especialmente oportuna en los países del sur de Europa, donde los efectos de la crisis fueron devastadores y parece que la recuperación no está teniendo el mismo efecto para todos los hogares. En el momento de redactar este artículo, estos hallazgos cobran aún más importancia, si cabe, dadas las graves consecuencias sobre el empleo de una crisis sanitaria que agravará la precariedad y desigualdad de las familias según el tipo de hogar, sumándose a los efectos de la crisis anterior. Los resultados aquí presentados alertan, por tanto, sobre la necesidad de diseñar políticas familiares y laborales acordes con el significado y alcance del cambio familiar y la relevancia de la estructura familiar en el contexto de la STD, lo que exige activar mecanismos de inclusión para niños, jóvenes y madres en situación de desventaja económica, formativa y laboral. En suma, estos resultados tienen importantes implicaciones teóricas y empíricas, además de evidenciar la relevancia que tiene el hogar para explicar la desigualdad laboral, económica e intergeneracional, así como en la explicación de la pobreza y el bienestar infantiles.

\section{AGRADECIMIENTOS}

Este artículo se ha desarrollado dentro del proyecto "El bienestar infantil y la privación material ante los nuevos escenarios familiares de la precariedad en España" (RTI2018-099666-B-I00), financiado por el Ministerio de Ciencia, Innovación y Universidades. Además, los autores quieren agradecer la valiosa ayuda prestada por Anna Garriga Alsina.

\section{REFERENCIAS BIBLIOGRÁFICAS}

Alamillo, J. 2016. "Family Structure and Reproduction of Inequality: A Decomposition Approach". Working Paper N. ${ }^{\circ}$ 49. Mathematica Policy Research. Chicago.

Amato, P. R., A. Booth, S. M. McHale y J. V. Hook. eds. 2015. Families in an Era of Increasing Inequality: Diverging Destinies. Cham: Springer International Publishing.

Amato, P. R. y F. Fowler. 2002. "Parenting Practices, Child Adjustment, and Family Diversity". Journal of Marriage and Family 64(3): 703-16. https://doi.org/10.1111/ j.1741-3737.2002.00703.x.

Aysa-Lastra, M. y L. Cachón. 2015. "Unemployment and Nonstandard Employment Among Natives and Latinos in the US and Spain". Pp. 47-65 en Immigrant Vulnerability and Resilience: Comparative Perspectives on Latin American Immigrants During the Great Recession, editado por M. Aysa-Lastra y L. Cachón. Cham: Springer International Publishing. https://doi. org/10.1007/978-3-319-14797-0_3.

Baxter, J., M. Gray, K. Hand y A. Hayes. 2012. "Parental Joblessness, Financial Disadvantage and the Wellbeing of Parents and Children". SSRN Electronic Journal. Consulta 17 de junio del 2019 (https://ssrn.com/abstract $=2293760$ ).

Bell, D. y D. Blanchflower. 2011. "Young People and the Great Recession". Oxford Review of Economic Policy 27(2):
241-267. https://doi.org/10.1093/oxrep/grr011.

Bernardi, F. y J. Radl. 2014. "The Long-Term Consequences of Parental Divorce for Children's Educational Attainment". Demographic Research 30: 1653-1680. https:// doi.org/10.4054/DemRes.2014.30.61.

Boertien, D., F. Bernardi y J. Härkönen. 2018. "Family Structure and Socioeconomic Inequality of Opportunity in Europe and the United States". Pp. 165-178 en Unequal Family Lives: Causes and Consequences in Europe and the Americas, editado por N.R. Cahn, J. Carbone, L.F. DeRose y W. B. Wilcox. Cambridge: Cambridge University Press.

Brady, D. y R. Burroway. 2012. "Targeting, Universalism, and Single-Mother Poverty: A Multilevel Analysis Across 18 Affluent Democracies". Demography 49(2): 719-746. https://doi.org/10.1007/s13524-012-0094-z.

Brand, J. E. y J. Simon Thomas. 2014. “Job Displacement among Single Mothers: Effects on Children's Outcomes in Young Adulthood". American Journal of Sociology 119(4): 955-1001. https://doi.org/10.1086/675409.

Brown, S. L., W. D. Manning y J. B. Stykes. 2015. "Family Structure and Child Well-Being: Integrating Family Complexity". Journal of Marriage and Family 77(1): 177-90. https://doi.org/10.1111/jomf.12145.

Cahn, N. R., J. Carbone, L. F., DeRose y W. B. Wilcox. eds. 2018. Unequal Family Lives: Causes and Consequences in Europe and the Americas. Cambridge: Cambridge University Press.

Cantillon, B., Y. Chzhen, S. Handa y B. Nolan. eds. 2017. Children of Austerity: Impact of the Great Recession on Child Poverty in Rich Countries. Oxford: Oxford University Press.

Chzhen, Y. 2017. "Unemployment, Social Protection Spending and Child Poverty in the European Union during the Great Recession". Journal of European Social Policy 27(2): 123-37. https://doi.org/10.1177/0958928716676549.

Chzhen, Y. y J. Bradshaw. 2012. "Lone Parents, Poverty and Policy in the European Union". Journal of European Social Policy 22(5): 487-506. https://doi. org/10.1177/0958928712456578.

Cohen, P. N. 2015. "Divergent Responses to Family Inequality". Pp. 25-33 en Families in an Era of Increasing Inequality: Diverging Destinies, National Symposium on Family Issues, editado por P. R. Amato, A. Booth, S. M. McHale y J. Van Hook. Cham: Springer International Publishing.

Dannefer, D. 2003. "Cumulative Advantage/Disadvantage and the Life Course: Cross-Fertilizing Age and Social Science Theory". The Journals of Gerontology Series B 58(6): S327-S337. https://doi.org/10.1093/geronb/58.6.S327.

Duncan, G. J., K. M. Ziol-Guest y A. Kalil. 2010. "Early-Childhood Poverty and Adult Attainment, Behavior, and Health". Child Development 81(1): 306-25. https://doi. org/10.1111/j.1467-8624.2009.01396.x.

Eamon, M. K. y C. Wu. 2013. "Employment, Economic Hardship, and Sources of Assistance in Low-Income, Single-Mother Families Before Versus During and After the Great Recession". Journal of Poverty 17(2): 135156. https://doi.org/10.1080/10875549.2013.775995.

Erman, J. y J. Härkönen. 2017. "Parental Separation and School Performance Among Children of Immigrant Mothers in Sweden". European Journal of Population 33(2): 26792. https://doi.org/10.1007/s10680-017-9419-3.

Esping-Andersen, G. 2007. "Sociological Explanations of Changing Income Distributions". American Behavioral Scientist 
50(5): 639-58. https://doi.org/10.1177/0002764206295011.

Esser, I. y K. Olsen. 2018. "Matched on job qualities? Single and coupled parents in European comparison". Pp. 285-310 en The triple bind of single-parent families, Resources, employment and policies to improve weII-being, editado por R. Nieuwenhuis y L.C. Maldonado. Bristol, UK; Chicago, IL, USA: Bristol University Press. https://doi.org/10.2307/j.ctt2204rvq.19.

Flaquer, L., A. Moreno Mínguez y T. Cano López. 2016. "Changing Family Models: Emerging New Opportunities for Fathers in Catalonia (Spain)?”. Pp. 65-81 en Balancing Work and Family in a Changing Society: The Fathers Perspective, Global Masculinities, editado por I. Crespi y E. Ruspini. New York: Palgrave Macmillan US. https://doi.org/10.1057/978-1-137-53354-8_5.

Garfinkel, I., S. McLanahan y C. Wimer. 2016. Children of the Great Recession. New York: Russell Sage Foundation.

Goldscheider, F., E. Bernhardt y T. Lappegård. 2015. "The Gender Revolution: A Framework for Understanding Changing Family and Demographic Behavior". Population and Development Review 41(2): 207-239. https:// doi.org/10.1111/j.1728-4457.2015.00045.x.

Härkönen, J. 2017. "Diverging destinies in international perspective: Education, single motherhood, and child poverty". Working Paper N. ${ }^{\circ}$ 713. Luxembourg Income Study (LIS). Luxembourg.

Härkönen, J. 2018. "Single-mother poverty: How much do educational differences in single motherhood matter?". Pp 31-50 en The triple bind of single-parent families, Resources, employment and policies to improve weIl-being, editado por R. Nieuwenhuis y L. C. Maldonado. Bristol, UK; Chicago, IL, USA: Bristol University Press. https://doi.org/10.2307/j.ctt2204rvq.8.

Härkönen, J., F. Bernardi y D. Boertien. 2017. "Family Dynamics and Child Outcomes: An Overview of Research and Open Questions". European Journal of Population, 33(2): 163-184. https://doi.org/10.1007/s10680017-9424-6.

Härkönen, J. y J. Dronkers. 2006. "Stability and Change in the Educational Gradient of Divorce. A Comparison of Seventeen Countries". European Sociological Review 22(5): 501-517. https://doi.org/10.1093/esr/jcl011.

Heckman, J. J. 2006. "Skill Formation and the Economics of Investing in Disadvantaged Children". Science 312(5782): 1900-1902. https://doi.org/10.1126/science. 1128898 .

Heinrich, C. J. 2014. "Parents' Employment and Children's Wellbeing". The Future of Children 24(1): 121-146. https://doi.org/10.1353/foc.2014.0000.

Horemans, J., I. Marx y B. Nolan. 2016. "Hanging in, but Only Just: Part-Time Employment and in-Work Poverty throughout the Crisis". IZA Journal of European Labor Studies 5(1). https://doi.org/10.1186/s40174-0160053-6.

Hrženjak, M. 2017. "A Qualitative Study of Labour Market Precarisation and Involved Fatherhood in Slovenia". Revija Za Sociologiju 47(2): 207-232. https://doi.org/10.5613/ rzs.47.2.4.

lacovou, M. 2013. Household Composition, Poverty and Hardship across Europe. Luxembourg: Publications Office of the European Union.

Instituto Nacional de Estadística. 2007. "Encuesta de Población Activa". Madrid: Instituto Nacional de Estadística. Consulta 6 de mayo de 2020 (http://www.ine.es)

Instituto Nacional de Estadística. 2013. "Encuesta de Población Activa”. Madrid: Instituto Nacional de Estadística.
Consulta 6 de mayo de 2020 (http://www.ine.es)

Instituto Nacional de Estadística. 2018. "Encuesta de Condiciones de Vida". Madrid: Instituto Nacional de Estadística. Consulta 15 de febrero de 2019 (http://www.ine.es)

Instituto Nacional de Estadística. 2019. "Encuesta Continua de Hogares". Madrid: Instituto Nacional de Estadística. Consulta 6 de mayo de 2020 (http://www.ine.es)

International Labour Organization. 2011. Spain. Quality jobs for a new economy. Geneva: ILO Publications.

International Labour Organization. 2012. World of Work Report 2012. Better jobs for a better economy. Geneva: ILO Publications.

Kollmeyer, C. 2013. "Family Structure, Female Employment, and National Income Inequality: A Cross-National Study of 16 Western Countries". European Sociological Review 29(4): 816-27. https://doi.org/10.1093/esr/jcs060.

Lamb, M. E. 1997. The role of the father in child development. New York: Wiley.

Lesthaeghe, R. 2010. "The Unfolding Story of the Second Demographic Transition". Population and Development Review 36(2): 211-251. https://doi.org/10.1111/j.17284457.2010.00328.x.

Madruga Torremocha, I. 2006. Monoparentalidad y política familiar: dilemas en torno a la madre cuidadora/madre trabajadora. Madrid: CIS.

Main, G. 2014. "Child Poverty and Children's Subjective WeII-Being". Child Indicators Research 7(3): 451-472. https://doi.org/10.1007/s12187-014-9237-7.

Maldonado, L. C. y R. Nieuwenhuis. 2015. "Family Policies and Single Parent Poverty in 18 OECD Countries, 1978-2008". Community, Work \& Family 18(4): 395415. https://doi.org/10.1080/13668803.2015.1080661.

Malo, M. A. 2015. Labour Market Measures in Spain 20082013: The Crisis and Beyond. Geneva: ILO.

Martínez, R. y C. Navarro. 2014. "Pobreza y privación: tendencias y determinantes". Documento de Trabajo del VII Informe sobre Desarrollo y Exclusión Social en España. Fundación FOESSA. Madrid.

Mattingly, M., K. Smith y J. Bean. 2011. "Unemployment in the Great Recession: single parents and men hit hard". The Carsey School of Public Policy at the Scholars' Repository 144. Consulta 12 de Junio de 2019 (https:// scholars.unh.edu/carsey/144).

Matysiak, A., M. Styrc y D. Vignoli. 2014. "The Educational Gradient in Marital Disruption: A Meta-Analysis of European Research Findings". Population Studies 68(2): 197-215. https://doi.org/10.1080/00324728.2013.856459.

Mclanahan, S. 2004. "Diverging Destinies: How Children Are Faring under the Second Demographic Transition". Demography 41(4): 607-627. https://doi.org/10.1353/ dem.2004.0033.

McLanahan, S. y C. Percheski. 2008. "Family Structure and the Reproduction of Inequalities". Annual Review of Sociology 34(1): 257-276. https://doi.org/10.1146/annurev.soc.34.040507.134549.

Miguélez, F. y P. López-Roldán. 2014. Crisis, empleo e inmigración en España. Un análisis de las trayectorias laborales. Bellaterra (Barcelona): Universitat Autònoma de Barcelona.

Mooi-Reci, I. y J. Muñoz-Comet. 2016. "The Great Recession and the Immigrant-Native Gap in Job Loss in the Spanish Labour Market". European Sociological Review 32(6): 730-751. https://doi.org/10.1093/esr/jcw031.

Moreno Mínguez, A. e I. Crespi. 2017. "Future perspectives on work and family dynamics in Southern Europe: 
The importance of culture and regional contexts". International Review of Sociology 27(3): 389-393. https://doi.org/10.1080/03906701.2017.1378153.

Nieuwenhuis, R. y L. C. Maldonado. 2018a. "Single-Parent Families and In-Work Poverty". Pp. 171-192 en Handbook of Research on In-Work Poverty, editado por H. Lohmann e I. Marx. Cheltenham: Edward Elgar Publishing.

Nieuwenhuis, R. y L. C. Maldonado. 2018b. The triple bind of single-parent families: Resources, employment and policies to improve wellbeing. Bristol, UK; Chicago, IL, USA: Bristol University Press.

OECD. 2018. OECD Employment Outlook 2018. Paris: OECD Publishing. https://doi.org/10.1787/empl_outlook-2018-en.

Ortiz García, P. 2014. "El trabajo a tiempo parcial. ¿Una alternativa para la mujer en tiempos de crisis?". Sociología del Trabajo 82: 73-92.

Pong, S., J. Dronkers y G. Hampden-Thompson. 2003. "Family Policies and Children's School Achievement in SingleVersus Two-Parent Families". Journal of Marriage and Family 65(3): 681-699. https://doi.org/10.1111/j.17413737.2003.00681.x.

Putnam, R. D. 2015. Our Kids: The American Dream in Crisis. New York: Simon \& Schuster.

Rafferty, A. y J. Wiggan. 2017. "The Time-Related Underemployment of Lone Parents during Welfare Reform, Recession and Austerity: A Challenge to In-Work Conditionality?". Social Policy \& Administration 51(3): 511538. https://doi.org/10.1111/spol.12190.

Rees, G., H. Goswami y J. Bradshaw. 2010. Developing an index of children's subjective well-being in England. London: The Children's Society.

Restrepo, B. J. 2016. "Parental Investment Responses to a Low Birth Weight Outcome: Who Compensates and Who Reinforces?". Journal of Population Economics 29(4): 969-689. https://doi.org/10.1007/s00148-0160590-3.

Rodríguez Hernández, J. E. 2018. "Factors Determining Labor Underutilization in Spain by Gender before and after the Economic Crisis". Economic and Industrial Democracy. https://doi.org/10.1177/0143831X17752266.

Rodríguez Sumaza, C. 2001. "La estrategia británica de apoyo a las familias monoparentales a través del empleo". Revista Internacional de Sociología 59(30): 209. https://doi.org/10.3989/ris.2001.i30.773.

Salladarré, F. y S. Hlaimi. 2014. "Women and Part-Time Work in Europe". International Labour Review 153(2): 293-310. https://doi.org/10.1111/j.1564-913X.2014.00205.x.

Sum, A. e I. Khatiwada. 2010. "The Nation's underemployed in the "Great Recession» of 2007-09". Monthly Labor Review 11: 3-15.

Thévenon, O. 2011. "Family Policies in OECD Countries: A Comparative Analysis". Population and Development Review 37(1): 57-87. https://doi.org/10.1111/j.17284457.2011.00390.x

Treanor, M. C. 2018. "Income poverty, material deprivation and lone parenthood". Pp. 81-100 en The triple bind of single-parent families, Resources, employment and policies to improve well-being, editado por R. Nieuwenhuis y L. C. Maldonado. Bristol, UK; Chicago, IL, USA: Bristol University Press. https://doi.org/10.2307/j. ctt2204rvq.10.

Vacas-Soriano, C. 2018. “The 'Great Recession' and Low Pay in Europe". European Journal of Industrial Relations 24(3): 205-220. https://doi.org/10.1177/0959680117715932.

Van De Kaa, D. J. 1987. "Europe's Second Demographic Transition". Population Bulletin 42(1): 1-59.

Van Gyes, G. y L. Szekér. 2013. Impact of the Crisis on Working Conditions in Europe. Dublin: European Foundation for the Improvement of Living and Working Conditions.

Verick, S. 2009. "Who Is Hit Hardest during a Financial Crisis? The Vulnerability of Young Men and Women to Unemployment in an Economic Downturn". IZA Discussion Paper N. ${ }^{\circ}$ 4359. Consulta 12 de Junio de 2019. (https://ssrn.com/abstract=1455521).

Wilson, K. R. y M. R. Prior. 2011. "Father Involvement and Child Well-Being". Journal of Paediatrics and Child Health 47(7): 405-407. https://doi.org/10.1111/j.14401754.2010.01770.x.

Zaidi, B. y S. P. Morgan. 2017. "The Second Demographic Transition Theory: A Review and Appraisal". Annual Review of Sociology 43(1): 473-492. https://doi. org/10.1146/annurev-soc-060116-053442.

\section{NOTAS}

[1] La elección de los años 2008 y 2013 se debe a que, en términos de crecimiento del PIB, son el primer y último año del periodo de crisis económica. Por otra parte, 2017 es el último año del periodo de recuperación para el que había datos disponibles en el momento de realización de los análisis.

[2] Cuando hacemos mención a "todos los padres del hogar", nos referimos al padre y a la madre de las familias biparentales y al padre o la madre de la familia monoparental.

[3] En los hogares monoparentales, la variable de intensidad laboral solo puede tomar los valores 0, 0.5 y 1.

[4] Adicionalmente, también se optó por utilizar una variable de bajos ingresos laborales netos. No obstante, los resultados obtenidos con esta variable son similares a los presentados en este artículo. Los resultados están disponibles bajo petición.

[5] Los ingresos se ajustaron según el tamaño del hogar utilizando la escala de equivalencia de la OCDE, lo cual, como señalan Nieuwenhuis y Maldonado (2018a), permite tener en cuenta la falta de un segundo padre en los hogares monoparentales.

[6] Las variables de ingresos de la ECV informan sobre los ingresos obtenidos por los individuos en el año anterior al de la entrevista, por lo que, en este caso, realmente se están comparando los años 2007, 2012 y 2016.

[7] Dadas las pocas observaciones de hogares monoparentales encabezados por hombres (176 observaciones), en los análisis no distinguimos entre estos y los encabezados por mujeres.

[8] En esta categoría también se incluyen los hogares en que los niños son hijos de un solo miembro de la pareja. 


\section{ANEXO}

Tabla A1.

Participación laboral en hogares biparentales, 2008- 2017

\begin{tabular}{|l|c|c|}
\hline & $\mathbf{2 0 0 8}$ & $\mathbf{2 0 1 3}$ \\
\hline Ambos padres trabajan (\% hogares biparentales) & 55.14 & 44.83 \\
\hline Solo uno de los padres trabaja (\% hogares biparentales) & 38.51 & 41.76 \\
\hline Ninguno de los padres trabaja (\% hogares biparentales) & 6.35 & 33.44 \\
\hline
\end{tabular}

Fuente: Elaboración propia a partir de las ECV de 2008, 2013 y 2017

Tabla A2.

Tasas de ocupación y trabajo a tiempo parcial en hogares monoparentales y biparentales, 2008- 2017

\begin{tabular}{|c|c|c|c|c|c|c|}
\hline & \multicolumn{3}{|c|}{ Tasa de ocupación } & \multicolumn{3}{|c|}{ Tasa de trabajo a tiempo parcial } \\
\hline & 2008 & 2013 & 2017 & 2008 & 2013 & 2017 \\
\hline Hogares monoparentales (\% padres) & 75.55 & 58.06 & 65.14 & 19.75 & 24.85 & 18.66 \\
\hline Hogares biparentales (\% padres) & 74.39 & 65.71 & 76.32 & 11.75 & 13.80 & 9.66 \\
\hline
\end{tabular}

Fuente: Elaboración propia a partir de las ECV de 2008, 2013 y 2017

ANTONIO LUIS PÉREZ CORRAL es licenciado en Economía por la Universidad de Granada y actualmente trabaja como investigador predoctoral FPI en el Departamento de Sociología y Trabajo Social de la Universidad de Valladolid. Su campo de investigación se centra en la pobreza laboral y en el análisis de las políticas de protección social.

ALMUDENA MORENO MíNGUEZ es doctora en Sociología por la Universidad Autónoma de Barcelona y profesora en el Departamento de Sociología y Trabajo Social de la Universidad de Valladolid. Es especialista en temas familiares, Estado de bienestar, políticas públicas e investigación comparativa sobre género, equilibrio laboral-familiar y bienestar infantil. Actualmente está involucrada en varios proyectos nacionales y europeos. 